\title{
Other Primary Malignancies Among Women With Adult-Type Ovarian Granulosa Cell Tumors
}

\section{Bryk, Saara}

2018-10

Bryk , S , Pukkala , E , Färkkilä , A , Heikinheimo , M , Unkila-Kallio , L \& Riska , A 2018 , ' Other Primary Malignancies Among Women With Adult-Type Ovarian Granulosa Cell Tumors ' , International Journal of Gynecological Cancer , vol. 28 , no. 8 , pp. 1529-1534 . https://doi.org/10.1097/IG

http://hdl.handle.net/10138/311747

https://doi.org/10.1097/IGC.0000000000001333

cc_by_nc

acceptedVersion

Downloaded from Helda, University of Helsinki institutional repository.

This is an electronic reprint of the original article.

This reprint may differ from the original in pagination and typographic detail.

Please cite the original version. 
1 Other Primary Malignancies Among Women With Adult-type Ovarian

2 Granulosa Cell Tumors

6 Markku Heikinheimo, MD, $\mathrm{PhD}^{2,5}$, Leila Unkila-Kallio, MD, $\mathrm{PhD}^{1}$, Annika Riska,

$7 \mathrm{MD}, \mathrm{PhD}^{1}$

8

91 Obstetrics and Gynecology, University of Helsinki and Helsinki University Hospital

102 Children's Hospital, University of Helsinki and Helsinki University Hospital

113 Finnish Cancer Registry, Institute for Statistical and Epidemiological Cancer

12 Research, Helsinki, Finland

134 Faculty of Social Sciences, University of Tampere, Tampere, Finland

145 Department of Pediatrics, Washington University School of Medicine, St Louis

15 Children's Hospital, St Louis, Missouri

17 Corresponding author

18 Saara Bryk, M.D., Ph.D.

19 Obstetrics and Gynecology, University of Helsinki and Helsinki University Hospital,

20 PO Box 140, 00290 Helsinki, Finland

21 e-mail: saara.bryk@hus.fi

22 Tel: +358503559848

23

24 Keywords Granulosa Cell Tumor, Sex Cord-Stromal Tumors, Second Primary

25 Malignancy, Second Primary Cancer, Ovarian Cancer Epidemiology

26

Word count 2,490

28

Acknowledgments We thank Tiina Hakanen for invaluable assistance with the cancer registry data. This study received funding from the Finnish Cancer Foundation and University of Helsinki Research Funds.

The authors declare no conflicts of interest. 
Abstract

3 Objective To determine the incidence of new primary malignancies after adult-type

4 granulosa cell tumor (AGCT), and the incidence of AGCT after breast and uterine

5 cancer using nationwide population-based registry data.

6 Methods We identified all patients diagnosed with AGCT in 1968-2013 from the

7 Finnish Cancer Registry ( $\mathrm{n}=986$ ). The number of subsequent primary malignancies

8 among women with AGCT, and the number of AGCTs in women with previous

9 breast or uterine cancer were compared with the expected number of cases, and

10 expressed as Standardized Incidence Ratios (SIRs).

11 Results There were 122 cases of subsequent cancers diagnosed at least six months 12 after the primary diagnosis of AGCT (SIR 1.09, 95\% CI 0.91-1.3). Particularly, the 13 observed number of cancers of the soft tissue (SIR 4.13, 95\% CI 1.33-12.8), thyroid 14 (SIR 3.42, 95\% CI 1.54-7.62), and leukemia (SIR 2.67, 95\% CI 0.98-5.82) exceeded 15 the number of expected cases. The SIR for breast cancers after AGCT was 1.26 (95\% 16 CI 0.92-1.73), and the SIR for AGCT after breast cancer 1.59 (95\% CI 1.04-2.29).

17 The risk for subsequent AGCT was more than two-fold in breast cancer patients less 18 than 50 years of age, and over 15 years after primary diagnosis.

19 Conclusions There is an increased risk for thyroid and soft tissue cancer as well as 20 leukemia after AGCT, which may be associated with late effects of carcinogenic 21 treatments and possibly shared risk factors. After breast cancer, the risk for AGCT 22 was higher, which may indicate shared hormonal etiology. 
Adult type-granulosa cell tumors of the ovary (AGCTs) account for $5 \%$ of all ovarian neoplasms, and constitute the majority of sex cord-stromal tumors ${ }^{1}$. The recently reported age-adjusted (World Standard) incidence rates (truncated to age categories 20 years or older) of AGCT average around 0.6-0.8/100,000 women, and

31 peak after menopause ${ }^{2}$. AGCTs are characterized by their estrogen-secreting ability, 32 although it has been estimated that approximately $30 \%$ of these tumors do not secrete 33 estradiol, likely due to lack of theca cells in the tumor stroma ${ }^{1}$. A single somatic point 34 mutation in the transcription factor FOXL2 (402C-G) is the pathognomonic molecular 35 feature for $\mathrm{AGCTs}^{3}$. Otherwise, the etiological factors remain unknown, although 36 some studies have suggested a possible hormonal background for these tumors $\mathrm{s}^{4,5}$. 37 According to current knowledge, there is no hereditary predisposition for the 38 development of AGCT.

The diagnosis is usually made at an early stage, partly due to symptoms 40 related to hormone secretion, and the disease tends to run an indolent course.

41 Excessive exposure to tumor-derived estrogen among these patients leads to an 42 increased risk of concomitant endometrial pathology and endometrial cancer ${ }^{6-9}$.

43 There are, however, only a few studies focusing on other primary malignancies in 44 women with AGCT 10, 11 . In general, the risk of other primary malignancies after 45 ovarian cancer is associated with either inherent genetic or lifestyle-related extrinsic 46 risk factors, or carcinogenic treatment regimens ${ }^{12-14}$. As other, particularly endocrine47 related cancers may share etiological factors with AGCTs, it is of interest to study the 48 potential association of these cancers, especially breast and uterine cancer. The object 
of our study was to evaluate the incidence of all other primary cancers after AGCT, as well the incidence of AGCT after breast or uterine cancer.

Materials and methods

In this retrospective cohort study, we identified all patients diagnosed with AGCT in Finland during 1968-2013 from the Finnish Cancer Registry (FCR). The FCR is a high-quality, population-based registry relying on unique personal identity codes. The personal identity code is a specific means of identification, which remains unchanged throughout the person's lifetime, and has been used in Finland since the 1960s. Physicians, hospitals, and pathology and hematology laboratories in Finland are obliged to report all malignant tumors to the FCR, resulting in a nearly complete

61 registration of all cancer cases ${ }^{15}$. Information on vital status and emigration was 62 obtained from the Population Register Center, which is directly linked to the FCR 63 information.

AGCTs were retrieved from the registry applying the ICD topography code 65 C56.9 with morphology codes M8620/1, 8620/3, 8621/1, and 8621/3. The incidence rates of AGCT during the follow-up period were calculated, and adjusted for age to the World Standard Population. All patients were followed up for second primary 68 cancer from the date of first diagnosis (1968-2013) to the date of death, date of 69 migration, or until December $31^{\text {st }}, 2013$. In order to identify concomitant cancers and 70 surveillance bias, the analyses were carried out in two subgroups: 1) all subsequent 71 tumors after AGCT, and 2) all subsequent tumors except those occurring within six 72 months after AGCT. Subsequent primary tumors were grouped in 18 categories based 73 on cancer site, and included ICD-codes C00-96, D32-33, D42-43, D45-47, and D76 
74 (mouth/pharynx, digestive organs, respiratory organs, breast, female genitalia, urinary

75 organs, melanoma of the skin, skin (other than melanoma), eye, thyroid gland, other

76 endocrine glands, bone, soft tissues, mesothelioma, autonomic nervous system,

77 brain/central nervous system, lymphoid/hematopoietic tissue, other/not defined). The

78 number of new primary malignant tumors among women with previous AGCT was

79 compared with the expected number of cases calculated from the accumulated person-

80 years and incidence rates for the national population, stratified by age and year of

81 diagnosis.

82 Secondly, we analyzed the number of subsequent AGCTs in women with a

83 first primary breast or uterine cancer (ICD C54 and C50), and compared it with the

84 expected number of AGCTs. These analyses were likewise performed separately on

85 all subsequent AGCTs as well as those occurring within 6 months of the primary

86 cancer diagnoses. The ratio of observed to expected cases was defined as the

87 Standardized Incidence Ratio (SIR), and 95\% confidence intervals (CI) were

88 calculated. The SIRs were also stratified for time since first primary cancer diagnosis

89 (0-4 years, 5-14 and 15+ years after the diagnosis of first primary tumor), for age at

90 the first primary cancer diagnosis ( $<50$ or 50 years or older), and in breast cancer also

91 for the invasion status (localized vs. non-localized).

92 The ethics committee of Helsinki University Hospital (HUH) and the National

93 Supervisory Authority for Welfare and Health approved the study.

94

95

96 Results

97 
In 1968-2013, a total of 986 women in Finland were diagnosed with AGCT.

99 The age-adjusted (World Standard) incidence varied between 0.4 and 0.9 per 100,000

100 women, with approximately 20 cases each year (Figure 1). The logarithmic trend line

101 suggests a decreasing trend in the incidence of AGCT over the 45-year study period.

102 After the diagnosis of AGCT, 122 cases of new primary malignant tumors

103 were recorded, resulting in a $12.4 \%$ rate of second malignancies among AGCT

104 patients. The expected number was 111.7 (SIR 1.09, 95\% CI 0.91-1.3) (Table 1). If

105 also cancers diagnosed within six months of AGCT were included, the total rate was

$10613.9 \%$ and SIR 1.19 (95\% CI 1-1.41, p=0.04). The SIR for these cancers only was 1075.00 (95\% CI 2.80-8.23). The median interval between the diagnosis of AGCT and 108 second primary tumor was 19.2 years (range $0.02-45.6$ years). In a minimum time of 109 six months from the primary cancer diagnosis, the observed number of thyroid cancer, 110 soft tissue cancer, and leukemia exceeded the number of expected cases significantly

111 (Table 1). The SIRs were also elevated for cancers of the oropharynx, breast, urinary 112 organs, skin (non-melanoma), and mesothelioma, but not significantly (Table 1).

113 There were less than expected cancer cases in the uterine corpus and ovaries. For all 114 subsequent cancers, the SIRs stratified for follow-up time were 0.75 for $0.5-4$ years 115 (95\% CI $0.45-1.15), 0.98$ for 5-14 years (95\% CI $0.72-1.30)$, and 1.40 (95\% CI $1.07-$ 116 1.78) for more than 15 years after diagnosis of AGCT. The SIR was higher for 117 patients who were less than 50 years of age at primary diagnosis (SIR 1.31, 95\% CI 118 0.96-1.75). The results were largely similar when also second primary cancer cases 119 diagnosed within six months after AGCT were included, with the exception of uterine 120 cancer.

121 The SIR for breast cancer after AGCT was 1.4 after at least five years of 122 primary cancer diagnosis (Table 2). Subsequent breast cancer was somewhat more 
123 common in patients who were at least 50 years old at the time of AGCT diagnosis

124 (SIR 1.31, 95\% CI 0.86-1.91). The SIR was only elevated in localized breast cancer

125 (SIR 1.36, 95\% CI 0.86-2.02), as opposed to non-localized breast cancer (SIR 0.83,

126 95\% CI 0.42-1.46). In patients who had breast cancer diagnosed primarily, there were

12725 cases of subsequent AGCTs during follow-up (Table 3). The SIR for AGCT after

128 breast cancer was 1.59 (95\% CI 1.04-2.29), and increased with time since breast 129 cancer diagnosis to 2.28 (95\% CI $0.98-4.41)$ in the follow-up category of 15 years or 130 more. For age below 50 years at breast cancer diagnosis the SIR was $2.10(95 \%$ CI $131 \quad 1.09-3.59)$.

132 From the cancers diagnosed within six months of AGCT, uterine cancer 133 accounted for $33 \%(n=5)$, digestive organs $27 \%(n=4)$, and breast cancer $13 \%(n=2)$ 134 of these cases. Other malignancies reported within this follow-up period included 135 cancers of the urinary tract, and lymphoid/hematopoietic tissue. After uterine cancer, 136 AGCT was diagnosed in 20 women within six months (SIR 4.99, 95\% CI 3.18-7.37), 137 whereas two women developed AGCT more than 6 months after primary uterine 138 cancer diagnosis. All women with uterine cancer and subsequent AGCT were at least 13950 years of age at the time of the uterine cancer diagnosis (SIR 6.21, 95\% CI 4.09140 9.42)

142 Discussion

The indolent course, relatively low disease-related mortality and estrogensecreting capability of AGCT result in a clinically relevant lifetime risk for

146 developing a second primary cancer. On the other hand, the etiological factors of 147 AGCT are largely unknown, and a common predisposing factor may exist behind 
148 AGCT and other hormone-related cancers. To our knowledge, this is the largest and 149 first study since Björkholm et al. in $1980^{11}$ to analyze all second primary malignancies 150 among AGCT patients. In our study, women with AGCT had a 9\% increased risk of 151 developing a new primary malignancy as compared with the general population. If 152 cancers diagnosed within six months after the primary tumor were included, the risk 153 was significantly increased by $19 \%$. The large difference in these figures is mainly 154 explained by the presence of concomitant endometrial cancer, but the significant 155 number of cancers of the digestive organs diagnosed within six months of AGCT 156 most likely also reflects the increased surveillance among cancer patients in general.

157 Two recent publications have described the incidence of endometrial cancer 158 and breast cancer among patients with AGCT ${ }^{6,10}$. Van Meurs et al. found a $6 \%$ rate 159 of endometrial cancer concomitant with the diagnosis of AGCT, but no increased risk 160 for endometrial abnormalities in the median follow-up time of 10 years after AGCT 161 for patients not having undergone hysterectomy ${ }^{6}$. Other population-based studies have 162 reported 5-8\% rates of concomitant endometrial cancer ${ }^{8,9,11}$, and we reported similar 163 rates in a large, single-institute patient cohort ${ }^{16}$. In the current population-based 164 registry cohort, the rate was $2.5 \%$ when patients diagnosed primarily with either 165 AGCT or uterine cancer and a subsequent uterine cancer or AGCT within six months 166 of primary diagnosis were included. This relatively low rate may reflect a proportion 167 of previously hysterectomized patients, since they could not be excluded from the 168 original cancer registry data. This would also explain the higher incidence in hospital169 based cohorts, as solely patients with endometrial sample available have been 170 evaluated.

171 We found an increased risk for breast cancer both before and after diagnosis of 172 AGCT, although the risk was significant only before AGCT. This is a similar finding 
173 to the smaller Danish, Israeli and US studies where the rate of breast cancer among

174 AGCT patients was 5-10\% ${ }^{9,10,17}$. In our study, the rate of breast cancer was $6.9 \%$

175 among all women with AGCT. After AGCT, the risk was confined to localized breast

176 cancers, which may indicate towards surveillance bias, i.e. the increased frequency

177 and intensity of clinical follow-up and examination among patients with previously

178 diagnosed cancer. There was a relatively long latency between breast cancer and

179 AGCT regardless of which cancer was the first primary tumor, which does not

180 support genetic susceptibility. AGCT is neither associated with any of the known

181 predisposing mutations to breast cancer such as BRCA1 and BRCA2 mutations, nor is

182 the FOXL2 mutation pathognomonic to AGCT present in breast carcinoma ${ }^{1,18}$. In

183 the present study, the risk for subsequent AGCT in breast cancer patients was

184 significantly increased in women who were younger than 50 years at primary

185 diagnosis, which probably reflects the long follow-up time, as the latency between the

186 cancers was also long. Shared etiological factors such as obesity, parity, and hormonal

187 environment offer a possible explanation for the increased incidence of breast cancer

188 and AGCT among same women. Obesity represents a hyperestrogenic state and is a

189 known risk factor for breast cancer $^{19}$, and has been suggested as a risk factor for

$190 \mathrm{AGCT}^{4}$. In post-menopausal women, breast cancer risk is around twice as high in

191 those with the highest sex hormone levels compared to those with the lowest ${ }^{20}$. Parity,

192 on the other hand, is a protective factor in both breast and ovarian cancer ${ }^{21,22}$.

193 The effects of primary cancer treatment may influence the development of

194 second primary AGCT. Selective estrogen receptor modulators (SERMs) such as

195 tamoxifen are used to treat hormone-receptor positive breast cancer, and three case

196 reports have linked antecedent tamoxifen use with the development of AGCT $^{23-25}$.

197 Furthermore, aromatase inhibitors such as letrozole are used in the treatment of both 
postmenopausal breast cancer and $\mathrm{AGCT}^{26,}{ }^{27}$. However, further evidence is warranted to establish a causal link between hormonal breast cancer treatment and the development of AGCT.

Similarly to an earlier study, we found an increased risk for thyroid cancer among patients with previous AGCT, but it should be noted that the number of cases 203 is rather small in both studies ${ }^{11}$. It has been proposed that female hormones, 204 reproductive factors, and obesity also play a role in thyroid cancer pathogenesis, but 205 there are no consistent data linking ovarian and thyroid cancer ${ }^{28,29}$. DICER1 germline 206 mutation carriers have a predisposition to both thyroid cancer and sex cord-stromal, 207 particularly Sertoli-Leydig cell tumors ${ }^{30,31}$. Cancer registry data are not, however, 208 molecularly validated, and there is a possibility that some of the tumors identified as 209 AGCT may actually represent other sex cord-stromal tumors.

210 We also found significantly increased SIRs for soft tissue cancer and leukemia 211 after AGCT. The development of secondary soft tissue sarcoma is strongly associated 212 with radiation exposure from radiotherapy, especially after breast cancer ${ }^{32,} 33$. No 213 association between female reproductive factors and the development of soft tissue 214 cancer has been detected, but the studies in this field are scarce ${ }^{34}$. As nowadays 215 radiotherapy is rarely used in the treatment of AGCT, the increased SIR for soft tissue 216 cancer is most likely related to shared risk factors. Furthermore, radiotherapy for 217 ovarian cancer is known to be associated with bladder carcinoma, but in our series, 218 the SIR for bladder cancer was not significantly elevated after AGCT ${ }^{35}$. Adjuvant 219 therapy is used in the management of metastatic or recurrent AGCT, and presently 220 consists of platinum-based chemotherapeutic agents or more recently, hormonal 221 treatments $^{26,36}$. Late effects of chemotherapy may include increased risk for leukemia, 222 and most likely explains the high incidence of this cancer among patients with 
223 previous $\mathrm{AGCT}^{12,14}$. Two large population-based studies on second malignancies

224 after ovarian cancer of any type both reported significantly elevated SIRs for cancers

225 of the colon, rectum, lung, breast, bladder, and thyroid, as well as for leukemia, and

226 the risk for subsequent cancer development was associated with older age, chemo-

227 and radiotherapy ${ }^{12,35}$.

228 This is the largest study to date in analyzing the risk for other primary

229 malignancies associated with AGCT. The strengths of this study are the reliable and

230 comprehensive cancer registry incidence data, and the long observation period of over

23140 years. The rarity and the lack of molecular validation of AGCT, as well as of

232 individual data such as parity, BMI, or use of hormonal therapies are limiting factors

233 in this analysis.

234 In conclusion, we found a slightly elevated risk for overall second 235 malignancy, particularly thyroid and soft tissue cancer, and leukemia. Partly these 236 excesses may result from carcinogenic treatments for AGCT. The increased incidence 237 of AGCT and breast cancer among the very same patients may indicate shared 238 hormonal etiology. Earlier studies have concluded that breast cancer patients have a 239 higher incidence of second primary ovarian cancer, particularly when diagnosed 240 before 50 years of age; this patient group might benefit from regular gynecological 241 surveillance ${ }^{37-39}$. This seems to be true also for AGCT after breast cancer, which 242 should be recognized in patient counseling and long-term clinical follow-up after the 243 primary tumor. 
250 Acknowledgements We thank Tiina Hakanen for invaluable assistance with the

251 cancer registry data. This study received funding from the Finnish Cancer Foundation 252 and University of Helsinki Research Funds.

253

254 The authors declare no conflict of interest.

255

256

257 
1. Schumer ST, Cannistra SA. Granulosa cell tumor of the ovary.Journal of clinical oncology : official journal of the American Society of Clinical Oncology. 2003;21(6):1180-9.

2. Bryk S, Pukkala E, Martinsen JI, et al. Incidence and occupational variation of ovarian granulosa cell tumours in Finland, Iceland, Norway and Sweden during 1953-2012: a longitudinal cohort study. BJOG. 2017;124(1):143-9. 3. Jamieson S, Butzow R, Andersson N, et al. The FOXL2 C134W mutation is characteristic of adult granulosa cell tumors of the ovary. Modern pathology : an official journal of the United States and Canadian Academy of Pathology, Inc. 2010;23(11):1477-85.
4. Boyce EA, Costaggini I, Vitonis A, et al. The epidemiology of ovarian granulosa cell tumors: a case-control study. Gynecol Oncol. 2009;115(2):221-5. 5. Chen T, Surcel HM, Lundin E, et al. Circulating sex steroids during pregnancy and maternal risk of non-epithelial ovarian cancer. Cancer epidemiology, biomarkers \& prevention : a publication of the American Association for Cancer Research, cosponsored by the American Society of Preventive Oncology. 2011;20(2):324-36.

6. van Meurs HS, Bleeker MC, van der Velden J, et al. The incidence of endometrial hyperplasia and cancer in 1031 patients with a granulosa cell tumor of the ovary: long-term follow-up in a population-based cohort study. Int J Gynecol Cancer. 2013;23(8):1417-22.

7. Ayhan A, Salman MC, Velipasaoglu M, et al. Prognostic factors in adult granulosa cell tumors of the ovary: a retrospective analysis of 80 cases. J Gynecol Oncol. 2009;20(3):158-63.

8. Unkila-Kallio L, Tiitinen A, Wahlstr $\sqrt{ } \partial \mathrm{m} \mathrm{T}$, et al. Reproductive features in women developing ovarian granulosa cell tumour at a fertile age. Hum Reprod. 2000;15(3):589-93.

9. Ohel G, Kaneti H, Schenker JG. Granulosa cell tumors in Israel: a study of 172 cases. Gynecol Oncol. 1983;15(2):278-86.

10. Hammer A, Lauszus FF, Petersen AC. Ovarian granulosa cell tumor and increased risk of breast cancer. Acta Obstet Gynecol Scand. 2013;92(12):1422-5. 11. Björkholm E, Silfverswärd C. Granulosa- and theca-cell tumors. Incidence and occurrence of second primary tumors. Acta Radiol Oncol. 1980;19(3):161-7. 12. Hung YP, Liu CJ, Hu YW, et al. Secondary Primary Malignancy Risk in Patients With Ovarian Cancer in Taiwan: A Nationwide Population-Based Study. Medicine. 2015;94(38):e1626. 13. Keum N, Greenwood DC, Lee DH, et al. Adult weight gain and adiposityrelated cancers: a dose-response meta-analysis of prospective observational studies. Journal of the National Cancer Institute. 2015;107(2). 14. Travis LB, Holowaty EJ, Bergfeldt K, et al. Risk of leukemia after platinumbased chemotherapy for ovarian cancer. N Engl J Med. 1999;340(5):351-7. 15. Pukkala E, Engholm G, Hojsgaard Schmidt LK, et al. Nordic Cancer Registries - an overview of their procedures and data comparability. Acta Oncol. 2017:1-16. 
16. Bryk S, Farkkila A, Butzow R, et al. Clinical characteristics and survival of patients with an adult-type ovarian granulosa cell tumor: a 56-year single-center

17. Meisel JL, Hyman DM, Jotwani A, et al. The role of systemic chemotherapy in the management of granulosa cell tumors. Gynecol Oncol. 2015;136(3):50511.

18. Shah SP, K $\sqrt{\partial b e l} \mathrm{M}$, Senz J, et al. Mutation of FOXL2 in granulosa-cell tumors of the ovary. NEngl J Med. 2009;360(26):2719-29.

19. Kyrgiou M, Kalliala I, Markozannes G, et al. Adiposity and cancer at major anatomical sites: umbrella review of the literature. BMJ. 2017;356:j477.

20. Key T, Appleby P, Barnes I, et al. Endogenous sex hormones and breast cancer in postmenopausal women: reanalysis of nine prospective studies. Journal of the National Cancer Institute. 2002;94(8):606-16.

21. Adami HO, Hsieh CC, Lambe M, et al. Parity, age at first childbirth, and risk of ovarian cancer. Lancet. 1994;344(8932):1250-4.

22. Layde PM, Webster LA, Baughman AL, et al. The independent associations of parity, age at first full term pregnancy, and duration of breastfeeding with the risk of breast cancer. Cancer and Steroid Hormone Study Group. Journal of clinical epidemiology. 1989;42(10):963-73.

23. Gherman RB, Parker MF, Macri CI. Granulosa cell tumor of the ovary associated with antecedent tamoxifen use. Obstetrics and gynecology. 1994;84(4 Pt 2):717-9.

24. Abahssain H, Kairouani M, Gherman R, et al. Granulosa cell tumor of the ovary and antecedent of adjuvant tamoxifen use for breast cancer. World journal of surgical oncology. 2010;8:67.

25. Tanaka T, Kato T, Ohmichi M. Granulosa cell tumor of the ovary after longterm use of tamoxifen and toremifene. The journal of obstetrics and gynaecology research. 2012;38(12):1379-84.

26. van Meurs HS, van Lonkhuijzen LR, Limpens J, et al. Hormone therapy in ovarian granulosa cell tumors: a systematic review. Gynecol Oncol. 2014;134(1):196-205.

27. Schiavon G, Smith IE. Status of adjuvant endocrine therapy for breast cancer. Breast cancer research : BCR. 2014;16(2):206.

28. Negri E, Dal Maso L, Ron E, et al. A pooled analysis of case-control studies of thyroid cancer. II. Menstrual and reproductive factors. Cancer Causes Control. 1999;10(2):143-55.

29. Zhu J, Zhu X, Tu C, et al. Parity and thyroid cancer risk: a meta-analysis of epidemiological studies. Cancer Med. 2016;5(4):739-52.

30. Rutter MM, Jha P, Schultz KA, et al. DICER1 Mutations and Differentiated Thyroid Carcinoma: Evidence of a Direct Association. J Clin Endocrinol Metab. 2016;101(1):1-5.

31. Stewart CJ, Charles A, Foulkes WD. Gynecologic Manifestations of the DICER1 Syndrome. Surgical pathology clinics. 2016;9(2):227-41.

32. Fletcher CD. The evolving classification of soft tissue tumours - an update based on the new 2013 WHO classification. Histopathology. 2014;64(1):2-11. 33. Virtanen A, Pukkala E, Auvinen A. Incidence of bone and soft tissue sarcoma after radiotherapy: a cohort study of 295,712 Finnish cancer patients. Int J Cancer. 2006;118(4):1017-21. 


\section{1}

34. Fioretti F, Tavani A, Gallus S, et al. Menstrual and reproductive factors and risk of soft tissue sarcomas. Cancer. 2000;88(4):786-9.

35. Travis LB, Curtis RE, Boice JD, Jr., et al. Second malignant neoplasms among long-term survivors of ovarian cancer. Cancer research. 1996;56(7):1564-70.

36. Savage P, Constenla D, Fisher C, et al. Granulosa cell tumours of the ovary: demographics, survival and the management of advanced disease. Clinical oncology. 1998;10(4):242-5. 37. Molina-Montes E, Pollan M, Payer T, et al. Risk of second primary cancer among women with breast cancer: a population-based study in Granada (Spain). Gynecol Oncol. 2013;130(2):340-5.

38. Kirova YM, De Rycke Y, Gambotti L, et al. Second malignancies after breast cancer: the impact of different treatment modalities. Br J Cancer. 2008;98(5):870-4. 39. Hall HI, Jamison P, Weir HK. Second primary ovarian cancer among women diagnosed previously with cancer. Cancer epidemiology, biomarkers \& prevention : a publication of the American Association for Cancer Research, cosponsored by the American Society of Preventive Oncology. 2001;10(9):995-9. 


\section{Figure legends}

377

378 Figure 1. Incidence of adult-type ovarian granulosa cell tumors (AGCTs) in Finland 379 in 1968-2013, with a logarithmic trend line (dotted).

380

381

382

383

384 
Table 1. Risk of subsequent primary malignancies among Finnish women with previous adult-type ovarian granulosa cell tumor (AGCT) in 1968-2013, by site.

\begin{tabular}{|c|c|c|c|c|c|}
\hline Second primary tumor site & Observed & Expected & SIR & $95 \% \mathrm{CI}$ & p-value \\
\hline All & 137 & 114.7 & 1.19 & $1-1.41$ & 0.04 \\
\hline All, diagnosis within 6 months of AGCT & 15 & 3.00 & 5.00 & $2.80-8.23$ & $<0.001$ \\
\hline All, diagnosis > 6 months after AGCT & 122 & 111.7 & 1.09 & $0.91-1.3$ & 0.33 \\
\hline Mouth, pharynx & 3 & 1.7 & 1.76 & $0.57-5.45$ & 0.33 \\
\hline Digestive organs & 24 & 25.7 & 0.93 & $0.63-1.39$ & 0.74 \\
\hline Respiratory organs & 5 & 6.3 & 0.79 & $0.33-1.90$ & 0.60 \\
\hline Skin, melanoma & 3 & 3.0 & 0.99 & $0.32-3.06$ & 0.98 \\
\hline Skin, non-melanoma & 9 & 4.8 & 1.86 & $0.97-3.58$ & 0.06 \\
\hline Soft tissues & 3 & 0.7 & 4.13 & $1.33-12.8$ & 0.01 \\
\hline Breast & 38 & 30.2 & 1.26 & $0.92-1.73$ & 0.15 \\
\hline Female genitalia & 5 & 14.3 & 0.35 & $0.15-0.84$ & 0.02 \\
\hline Corpus uteri & 0 & 7.1 & 0.00 & $0.00-0.52$ & 0.01 \\
\hline Ovary & 1 & 4.3 & 0.23 & $0.01-1.30$ & 0.18 \\
\hline Cervix uteri & 2 & 1.3 & 1.49 & $0.18-5.38$ & 0.89 \\
\hline Other & 2 & 1.5 & 1.38 & $0.17-4.98$ & 0.97 \\
\hline Urinary organs & 8 & 5.6 & 1.43 & $0.71-2.86$ & 0.31 \\
\hline Bladder and urinary tract & 3 & 2.4 & 1.27 & $0.26-3.73$ & 0.92 \\
\hline Brain, central nervous system & 3 & 3.9 & 0.76 & $0.25-2.36$ & 0.64 \\
\hline Thyroid gland & 6 & 1.8 & 3.42 & $1.54-7.62$ & 0.003 \\
\hline Lymphoid and hematopoietic tissue & 11 & 9.6 & 1.15 & $0.64-2.07$ & 0.65 \\
\hline Leukemia & 6 & 2.2 & 2.67 & $0.98-5.82$ & 0.03 \\
\hline Other or not defined & 3 & 3.4 & 0.88 & $0.28-2.72$ & 0.82 \\
\hline
\end{tabular}

$\mathrm{SIR}=$ standardized incidence ratio, $\mathrm{CI}=$ confidence interval. Sites with $<3$ observed cases are excluded, with the exception of cancers of the female genitalia. 
Table 2. Risk of subsequent breast cancer among Finnish women with previous adulttype granulosa cell tumor (AGCT) in 1968-2013, by age at and time since AGCT diagnosis, and breast cancer invasion.

\begin{tabular}{llllll} 
& Observed & Expected & SIR & 95\% CI & p-value \\
\hline All & 40 & 31 & 1.29 & $0.93-1.73$ & 0.11 \\
$\begin{array}{l}\text { All, diagnosis }>6 \text { months } \\
\text { after AGCT }\end{array}$ & 38 & 30.2 & 1.26 & $0.9-1.7$ & 0.15
\end{tabular}

Follow-up time (years)

$\begin{array}{llllll}0-4 & 6 & 6.9 & 0.87 & 0.34-1.76 & 0.73 \\ 5-14 & 17 & 12.3 & 1.38 & 0.82-2.15 & 0.18 \\ \geq 15 & 15 & 10.9 & 1.37 & 0.79-2.19 & 0.22\end{array}$

Age at AGCT diagnosis

$\begin{array}{llllll}<50 & 14 & 11.9 & 1.18 & 0.66-1.91 & 0.54 \\ \geq 50 & 24 & 18.3 & 1.31 & 0.86-1.91 & 0.18\end{array}$

Breast cancer invasion ${ }^{1}$

$\begin{array}{lccccc}\text { Localized } & 21 & 15.5 & 1.36 & 0.86-2.02 & 0.16 \\ \text { Non-localized } & 10 & 12.0 & 0.83 & 0.42-1.46 & 0.56\end{array}$

AGCT = adult-type ovarian granulosa cell tumor, $\mathrm{SIR}=$ standardized incidence ratio, $\mathrm{CI}$

$=$ confidence interval.

${ }^{1}$ Invasion status unknown in seven cases 
Table 3. Risk of subsequent adult-type granulosa cell tumors (AGCTs) among Finnish women with breast cancer in 1968-2013, by age at and time since breast cancer diagnosis.

Observed Expected SIR 95\% CI p-value

\begin{tabular}{llllll}
\hline All & 28 & 16.6 & 1.69 & $1.14-2.4$ & 0.006 \\
$\begin{array}{l}\text { All, diagnosis > 6 months after } \\
\text { breast cancer }\end{array}$ & 25 & 15.7 & 1.59 & $1.04-2.29$ & 0.02 \\
$\begin{array}{l}\text { Follow-up time (years) } \\
0-4\end{array}$ & & & & & \\
$5-14$ & 8 & 5.9 & 1.35 & $0.62-2.52$ & 0.39 \\
$\geq 15$ & 10 & 6.8 & 1.48 & $0.74-2.59$ & 0.22 \\
& 7 & 3.1 & 2.28 & $0.98-4.41$ & 0.03
\end{tabular}

Age at breast cancer diagnosis

$\begin{array}{llllll}<50 & 11 & 5.2 & 2.10 & 1.09-3.59 & 0.01 \\ \geq 50 & 14 & 10.5 & 1.33 & 0.75-2.16 & 0.28\end{array}$

$\mathrm{SIR}=$ standardized incidence ratio, $\mathrm{CI}=$ confidence interval. 


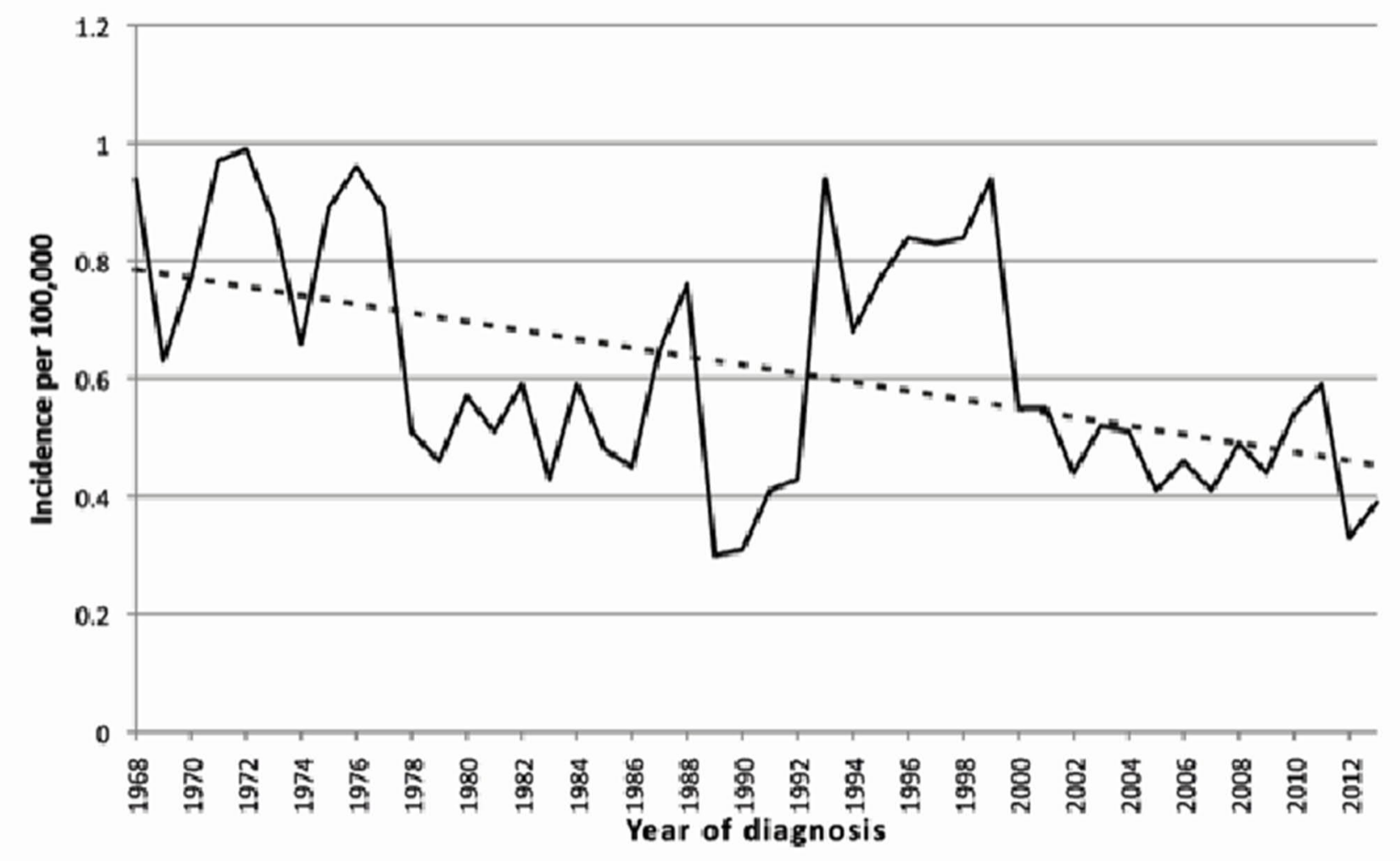

Figure 1. Incidence of adult-type ovarian granulosa cell tumors (AGCTs) in Finland in 1968-2013, with a logarithmic trend line (dotted). 